\title{
Exatecan Mesylate
}

National Cancer Institute

\section{Source}

National Cancer Institute. Exatecan Mesylate. NCI Thesaurus. Code C1679.

A semisynthetic, water-soluble derivative of camptothecin with antineoplastic activity.

Exatecan mesylate inhibits topoisomerase I activity by stabilizing the cleavable complex between topoisomerase I and DNA and inhibiting religation of DNA breaks, thereby inhibiting DNA replication and triggering apoptotic cell death. This agent does not require enzymatic activation and exhibits greater potency than camptothecin and other camptothecin analogues. ( $\mathrm{NCI04)}$ 